\title{
On the Efficiency and Complexity of Distributed Spectrum Allocation
}

\author{
Lili Cao and Haitao Zheng \\ Department of Computer Science \\ University of California, Santa Barbara, CA 93106 U.S.A \\ Email: \{lilicao,htzheng\}@cs.ucsb.edu
}

\begin{abstract}
We study the efficiency and complexity of a distributed spectrum allocation algorithm using explicit user coordination. Users self-organize into coordination groups and adjust spectrum assignments in each local group to approximate an optimal assignment. Compared to the conventional topologybased optimizations, local coordination can significantly reduce the computation and communication overhead required to adapt to topology variations. We focus on analyzing the end-user performance in terms of spectrum efficiency and communication overhead. We derive a theoretical lower bound on the amount of spectrum each user can get from coordination, and a theoretical upper bound on the algorithm convergence time. We also perform experiments to verify our analytical results.
\end{abstract}

\section{INTRODUCTION}

Wireless spectrum is a finite and scarce resource. To achieve efficient spectrum usage, future wireless devices will use cognitive radios [8], [15] to dynamically access locally available spectrum. Many have advocated for Open Spectrum Systems [1], [13] where cognitive radios become secondary devices and opportunistically access unused licensed spectrum, all without disrupting operations of any existing license owners, i.e. primary users.

The key challenge in dynamic spectrum access networks is how to maintain efficient spectrum sharing among (secondary) users. We hereby refer to secondary users as users. While maximizing spectrum utilization is the primary goal, we also need a good sharing mechanism to provide fairness across users. A user seizing spectrum without coordinating with others can cause harmful interference to its neighbors and hence reduce spectrum utilization.

Design of dynamic spectrum access networks can be divided into two types: power based spectrum sharing where users use the same spectrum band but transmit at different powers to minimize interference [6], [9], [10]; and channel sharing where users transmit at orthogonal channels to avoid interference. The mechanisms for channel sharing can be further divided into two categories.

(1) System-utility driven - Users share spectrum to maximize a predefined system utility [2], [3], [4], [11], [18], [19], [22]. This type of sharing also refers to collaborative sharing where users have signed agreements or are deployed by the same service providers to maximize system wide performance regardless of individual benefits.

(2) Self-gain driven - Users share spectrum to maximize self-benefit. Existing works have applied game theory to design and analyze spectrum usage strategies [16], [17]. This type of sharing refers to non-collaborative sharing where users are rational (and competitive), and are only interested in maximizing self-benefits.

In this paper, we focus on the system-utility driven, collaborative spectrum sharing. A motivating scenario is spectrum sharing among WiMAX/WiFi access points in order to support their subscribers. Examples include Googlenet in San Francisco and many other community networks. In this case, access points must share spectrum to avoid interference and maintain reliable communication links, while maximizing a predefined system utility.

The problem of spectrum management can be reduced into a variant of the graph coloring problem [3], [18], which is NP-hard. Given a small fixed network topology, existing approaches have proposed good heuristics based on centralized systems to obtain conflict free spectrum assignments that closely approximate the global optimum. In this case, a server collects information of user demand and assigns spectrum to a limited number of users to maximize system utility. However, when network topology, user demand and available spectrum at users change, the system needs to completely recompute spectrum assignments for all users after each change, resulting in high computational and communication overhead. This costly operation needs to be repeated frequently to maintain utilization and fairness.

In this paper, we consider a distributed approach to spectrum allocation that can potentially address the coexistence of a large number of users, while quickly adapting to network dynamics. Our previous work [4] proposes a distributed coordination algorithm where users affected by network dynamics selforganize into coordination groups and adapt their spectrum usage to approximate the new optimal conflict free assignment. While the work in [4] focuses on algorithm design, in this paper we propose to study end-user performance analytically in terms of spectrum efficiency and communication overhead. In particular, we derive a theoretical lower bound on the minimum amount of spectrum each user can get from coordination, and a theoretical upper bound of the algorithm convergence time. We then perform network simulations to verify our analytical results under different network settings.

The key contributions of this paper are two-fold:

Analytical Bounds on Distributed Coordination Performance and Complexity. We derive a theoretical lower 
bound on the amount of spectrum each user can get from the proposed distributed coordination, referred to as Poverty Line. This lower bound reflects the level of fairness enforced by the coordination algorithm, and can serve as a guidance for coordination. Further we derive an upper bound on the coordination complexity and overhead.

Simulation of Efficiency and Complexity. We conduct extensive simulations to quantify the performance of local coordination. Results indicate the proposed coordination performs similarly to the graph-coloring solution [23], [18] but with significantly reduced algorithm complexity. We also validate the poverty line and coordination overhead under different network settings, and study the impact of primary users, heterogeneous channel characteristics, and network topology.

\section{The Problem of Spectrum Allocation}

As background, we describe in this section our previous work on efficient and globally optimized spectrum allocation. We start with the theoretical model used to represent the general allocation problem. We describe how to reduce the optimal allocation problem to a variant of a graph multicoloring problem and describe previous solutions.

\section{A. Problem Model and Utility Functions}

We consider the case where the collection of available spectrum ranges forms a spectrum pool, divided into nonoverlapping orthogonal channels. We assume a network of $N$ users indexed from 0 to $N-1$ competing for $M$ spectrum channels indexed 0 to $M-1$. Each user can be a transmission link or a broadcast access point. Users select communication channels and adjust transmit power accordingly to avoid interfering with primaries. The channel availability and throughput for each user can be calculated based on the location and the channel usage of nearby primaries. The spectrum access problem becomes a channel allocation problem, i.e. to obtain a conflict free channel assignment for each user that maximizes system utility.

System utility. In this paper, we assign spectrum to users to maximize the following fairness based utility function:

$$
U(A)=\sum_{n=0}^{N-1} \log R_{A}(n)=\sum_{n=0}^{N-1} \log \sum_{m=0}^{M-1} a_{m, n} \cdot b_{m, n} .
$$

where $A=\left\{a_{m, n}, 0 \leq m \leq M-1,0 \leq n \leq N-1\right\}$ denotes a spectrum allocation, $a_{m, n}=1$ denotes that spectrum band $m$ is assigned to user $n$, and zero otherwise, and $b_{m, n}$ represents the maximum bandwidth/throughput that user $n$ can acquire through using spectrum band $m$ (assuming no interference from other neighbors).

Conflict graphs. Conflict graphs have been widely used to characterize interference constraints [3], [12], [14], [18], [20], [19]. We start from an illustrative example in Figure 1 where users $A$ to $E$ are for example access points that provide network access for their subscribers. Since $A, B$ and $C$ are located closely to each other, their subscribers will receive signals from all three APs. Signals from non-associated APs are

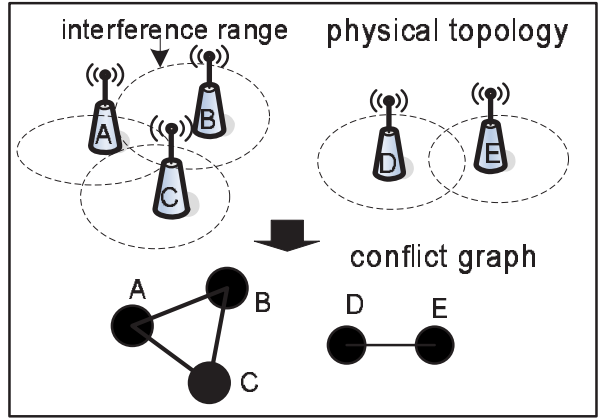

Fig. 1. A sample conflict graph.

interference that could disrupt communications. To minimize interference, $A, B$ and $C$ should not use the same spectrum concurrently. Those interference constraints can be modeled by a conflict graph $G=(V, L)$. Each vertex $v \in V$ represents a user and an edge $l(u, v) \in L$ exists between any two users if their communications interfere. Any two connected vertices should not use the same spectrum concurrently. The example of Figure 1 reduces to one triangle $(\mathrm{A}, \mathrm{B}, \mathrm{C})$ and one link $(\mathrm{D}, \mathrm{E})$.

The corresponding spectrum management problem is to color each vertex using a number of colors from its color list, and find the color assignment that maximizes system utility. The coloring is constrained by that if an edge exists between any two distinct vertices, they can't be colored with the same color. Most importantly, the objective of coloring is to maximize system utility. This is different from traditional graph coloring solutions that assign one color per vertex. Notice that the solution to this graph coloring problem is to maximize system utility for a given graph, i.e. a given topology and channel availability. This characterizes the optimal solution for a static environment.

\section{B. Existing Spectrum Management Solutions}

The optimal coloring problem is known to be NP-hard [7]. Existing efforts lead to efficient algorithms that closely approximate the optimum spectrum allocation for a given network topology. There are multiple complementary ways of addressing the problem of spectrum management, each applicable to different scenarios.

The work in [11] proposed a demand responsive pricing framework to use iterative bidding to maximize the expected revenue. They use exhaustive search to derive the optimal channel allocation for a small number of users. The work in [3], [2], [19] proposed the use of regional server model, and developed several heuristics based centralized approximations for a limited number of users. Results in [18], [23] show that the heuristic based algorithms perform similarly to the global optimum (derived off-line for simple topologies), and the centralized and distributed algorithms perform similarly. Further, the work in [21] proposed a hybrid pricing model - use simple auctions during peak period with a reserved price while applying a uniform price to all buyers during off-peak. Finally, another approach uses power based allocations and applies 


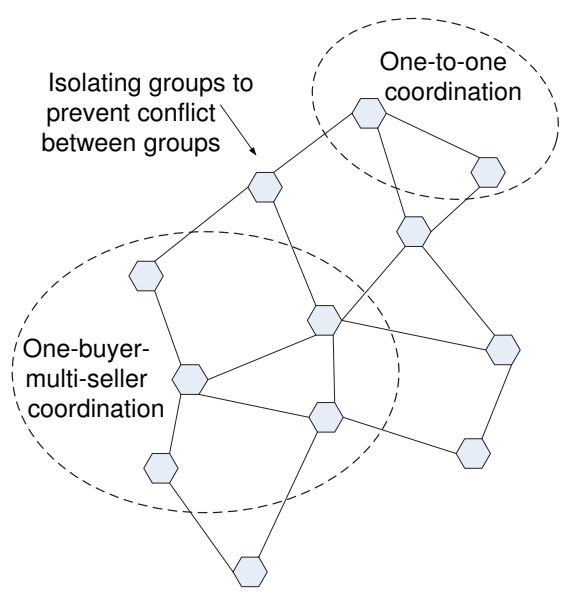

Fig. 2. An example of Coordination Groups.

price-driven power control to minimize interference [9]. In this case, all buyers use the same spectrum band.

\section{Distributed COORDination Framework}

In [4] we propose a distributed spectrum allocation framework using explicit coordination. Coordination involves sets of neighboring nodes (vertices in the conflict graph), each of which form a connected component of the conflict graph, self-organize into coordination groups. Each group modifies spectrum assignment within the group to improve system utility while ensuring that the change in spectrum assignment does not require any change at other nodes outside the group (due to interference constraints).

To initiate a coordination, any node who wants to improve its spectrum assignment first broadcasts a request to its neighbors who are in the interference range. These neighbors are connected to the node in the conflict graph. Neighbors willing to participate reply to the requester and form a coordination group. Because any two connected nodes in a conflict graph may not communicate directly, they can exchange coordination messages via multi-hop routing, or by using a dedicated control channel with higher power and hence larger transmission range.

\section{A. Regulated Coordination for Fast System Convergence}

To perform coordination, we must first determine the size and membership of distributed coordination groups. Large groups increase the complexity of coordination due to high synchronization and communication costs. In addition, interactions might occur between coordination groups if they share neighboring users. Careless coordination will lead to conflicts among concurrent coordination groups, and cascading effects that prevent system from converging. To facilitate coordination, we propose several constraints to regulate the procedure and avoid cascading effects that lead to system instability [4].

First, we limit the size of coordination groups to oneto-one coordination and one-buyer-multi-seller coordination. In this case, coordinating around a central leader per group

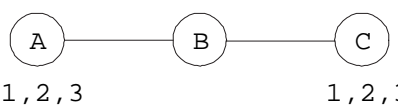

Fig. 3. An example of Starvation

can greatly simplify spectrum assignment. For each coordination group, the requester becomes the group coordinator and performs the coordination computation. Fig. 2 illustrates an example with one buyer and four sellers, and an one-to-one coordination.

We have shown in [4] that the effectiveness of one-toone coordination is limited by the number of exchangeable channels, and it can lead to node starvation. We then develop a special case of one-buyer-multi-seller coordination, referred to as Feed Poverty to eliminate node starvation. Figure 3 illustrates an example where the conflict graph is a chain topology consisting of three nodes A, B, and C. Node B is not assigned with any channel and the system utility is zero. We refer to this as user starvation. Node A and B cannot modify their spectrum usages due to the constraint from $\mathrm{C}$, while node $\mathrm{B}$ and $\mathrm{C}$ also cannot coordinate due to the constraint from A. However, by allowing $\mathrm{A}$ and $\mathrm{C}$ to give up channel 1 at the same time and feed it to B, we can remove the starvation at B. To achieve this, we propose a special one-buyer-multi-seller coordination, called Feed Poverty where if a node (buyer) has very poor channel assignment, the neighboring nodes can collaborate together to feed it with some channels.

Next, we limit the coordination to be self-contained. In particular, coordination in each group will not disturb the spectrum assignment at nodes outside the group. Hence, after the coordination, the modified channel assignment should not lead to any conflict with nodes outside the group. This helps to maintain system stability, so that a coordination may not invoke a series of reactions due to violations in interference constraints. More importantly, this guarantees that if a coordination improves the utility in a local area, it also improves the system utility. Or in other words, a local improvement will lead to a system improvement. We enforce this constraint by restricting exchangeable channels, and isolating any concurrent coordination groups. In particular, each node can only participate in at most one coordination group at any time, and the members of any two coordination groups can not be directly connected. Fig. 2 shows an example of isolation between coordination groups. Details of coordination process can be found in [4]. It should be noted that while the coordination algorithm is simple, implementing it in real systems requires an efficient protocol to form groups and exchange information. This is an important topic and we plan to address it in a future study.

\section{B. FC-Optimal Assignment}

We combine one-to-one Fairness Coordination and Feed Poverty Coordination into a Fairness Coordination with Feed Poverty (FC). Each node who wants to improve its spectrum usage starts with one-to-one Fairness Coordination with its 
neighbors to improve system utility. If there is no exchangeable channels, a poor node can broadcast a Feed-Poverty request to its neighbors to initialize Feed Poverty Coordination. Overall, a channel assignment $A$ is said to be $F C$-optimal if no further coordination can improve system utility.

\section{Theoretical Analysis}

In this section, we perform theoretical analysis on the proposed coordination approach. We examine system utility, user throughput and algorithm complexity for FC-optimal assignments. The analytical results provide insights to system fairness and spectrum utilization.

\section{A. Lower Bound on User Throughput}

We first examine the user throughput under any FC-optimal assignment, i.e. the user spectrum assignment when the system stabilizes and no one modifies its assignment. When the goal of optimization is to maximize system proportional fairness, we show that each user's throughput is lower-bounded.

Theorem 1: Under a FC-optimal assignment $A$, for each vertex $n$ in the conflict graph $G, 0 \leq n \leq N-1$, its throughput $R(n)$ is lower-bounded:

$$
\begin{aligned}
R(n) & >\frac{B(n)}{d(n)+1}-M B(n) \\
B(n) & \triangleq \sum_{m=0}^{M-1} b_{m, n} \\
M B(n) & \triangleq \max _{m=0}^{M-1} b_{m, n}
\end{aligned}
$$

where $d(n)$ represents the number of conflicting neighbors of $n$ or the degree of $n$ in the conflict graph, $B(n)$ represents $n$ 's total available bandwidth, and $M B(n)$ represents user $n$ 's maximum channel bandwidth across all the channels.

Proof: We prove the theorem by contradiction, that is we assume the bound (2) doesn't hold for node $n$ under a FCoptimal assignment, i.e.

$$
R(n) \leq \frac{B(n)}{d(n)+1}-M B(n) .
$$

Next, we will show that user $n$ can request a Feed Poverty coordination to further improve the system utility, which contradicts with the assumption that assignment $A$ is FCoptimal. The proof consists of two steps. We start from the procedure on finding the proper channel(s) to feed user $n$, and then prove that such feeding will increase system utility.

- Step 1: Select channel(s) to feed user $n$. We start from the following notations:

- User $n$ 's conflicting neighbors are indexed as user $\{0,1, \cdots, d(n)-1\}$.

- The channels assigned to user $n f_{A}(n)$ are indexed by $\left\{M-1, M-2, \cdots, M-\left|f_{A}(n)\right|\right\}$.

- $t \triangleq M-\left|f_{A}(n)\right|$ represents the number of channels not assigned to $n$.

- $d$ represents the number of non-starved neighbors of $n$, $d \leq d(n)$. A user with non-empty spectrum assignment is non-starved. We re-index these neighbors as $(0, \cdots, d-$ $1)$.

For a user $n$, we can represent the spectrum assignment matrix $A$ using the following table, where an element is 1 if the channel indexed by the row number is assigned to the user indexed by the column number.

\begin{tabular}{c|ccccc} 
Index & 0 & $\cdots$ & $\mathrm{d}(\mathrm{n})-1$ & $\mathrm{n}$ & $\cdots$ \\
\hline 0 & $\cdots$ & $\cdots$ & $\cdots$ & 0 & $\cdots$ \\
$\vdots$ & $\vdots$ & $\ddots$ & $\vdots$ & $\cdots$ & $\vdots$ \\
$t-1$ & $\cdots$ & $\cdots$ & $\cdots$ & 0 & $\cdots$ \\
$t$ & 0 & $\cdots$ & 0 & 1 & $\cdots$ \\
$\vdots$ & $\vdots$ & $\ddots$ & $\vdots$ & $\vdots$ & $\vdots$ \\
$\mathrm{M}-1$ & 0 & $\cdots$ & 0 & 1 & $\cdots$
\end{tabular}

We only focus on the first $t$ rows and first $d(n)+1$ columns (i.e. column $0,1, \cdots, d(n)-1, n$ ) since they represent the channel assignment at user $n$ 's conflicting neighbors. From (3) we can derive

$$
R(n) \leq \frac{B(n)}{d(n)+1}-M B(n) \leq \frac{B(n)}{d+1}-M B(n) .
$$

Integrating $A$ with channel bandwidth matrix $B$, we now construct an auxiliary matrix $R$ with $t$ rows and $d+1$ columns. The element of $R$ can be represented as

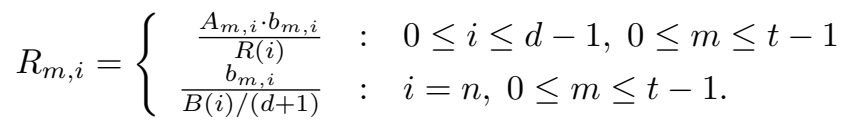

It is straightforward to show that for each column $0 \leq i \leq$ $d-1$,

$$
\sum_{m=0}^{t-1} R_{m, i}=\sum_{m=0}^{t-1} \frac{A_{m, i} \cdot b_{m, i}}{R(i)}=1 .
$$

and

$$
\sum_{m=0}^{t-1} \sum_{i=0}^{d-1} R_{m, i}=\sum_{i=0}^{d-1} \sum_{m=0}^{t-1} R_{m, i}=d .
$$

Further, we can show that for column $n$,

$$
\begin{aligned}
\sum_{m=0}^{t-1} R_{m, n} & =\sum_{m=0}^{t-1} \frac{b_{m, n}}{B(n) /(d+1)} \\
& =\frac{\sum_{m=0}^{t-1} b_{m, n}}{B(n) /(d+1)} \\
& =\frac{B(n)-R(n)}{B(n) /(d+1)} \\
(b y(4)) & \geq \frac{B(n)-\left(\frac{B(n)}{d+1}-M B(n)\right)}{B(n) /(d+1)} \\
& >d .
\end{aligned}
$$

Combining (6) and (7), we get

$$
\sum_{m=0}^{t-1} R_{m, n}>\sum_{m=0}^{t-1} \sum_{i=0}^{d-1} R_{m, i}
$$


Therefore, there must exist a channel $m_{0}$, s.t.

$$
R_{m_{0}, n}>\sum_{i=0}^{d-1} R_{m_{0}, i}
$$

Next we show that we can improve system utility by making user 0 to $d-1$ to give up channel $m_{0}$ and give this channel to user $n$.

- Step 2: Verify system utility improvement. From (3), we show that

$$
\begin{aligned}
& \frac{B(n)}{d+1}-M B(n) \geq R(n) \geq 0 \\
\Longrightarrow & \frac{B(n)}{d+1} \geq M B(n) \geq b_{m_{0}, n} \\
\Longrightarrow & R_{m_{0}, n}=\frac{b_{m_{0}, n}}{B(n) /(d+1)} \leq 1
\end{aligned}
$$

Combining (9) with (8), we have

$$
\begin{aligned}
\sum_{i=0}^{d-1} R_{m_{0}, i} & <1, \text { and } \\
R_{m_{0}, i} & <1, \quad 0 \leq i \leq d-1 .
\end{aligned}
$$

This demonstrates that $m_{0}$ is not the only channel assigned to each neighbor $i$. Hence, after giving up channel $m_{0}$ to user $n$, every neighbor has at least one additional channel remains.

Next, we need to compute the system utility after user $n$ is being fed by channel $m_{0}$, which depends on the spectrum assignment before the feeding. There are two possibilities:

- Type 1: $R(n)=0$. From (10), the feeding will increase $R(n)$ to $b_{m 0, n}$ without starving any neighbor ( 0 to $\left.d-1\right)$. Clearly, the system utility is improved from $-\infty$ if $n$ is the only starved user in the network before the feeding. However, when there are multiple starved users, the system utility remains $-\infty$. In this case, we modify the definition of improving system utility to include both reducing the number of starved users and increasing the fairness utility of non-starved users. Through a similar process, we can continue the remove other starved users and further improve system utility.

- Type 2: $R(n)>0$. The ratio of system utility after the feeding to that before the feeding can be computed as

$$
\begin{aligned}
G_{F P} & =\frac{\left(R(n)+b_{m_{0}, n}\right) \prod_{i=0}^{d-1}\left(R(i)-A_{m_{0}, i} b_{m_{0}, i}\right)}{R(n) \cdot \prod_{i=0}^{d-1} R(i)} \\
& =\frac{R(n)+b_{m_{0}, n}}{R(n)}\left(\prod_{i=0}^{d-1} \frac{R(i)-A_{m_{0}, i} b_{m_{0}, i}}{R(i)}\right) \\
& =\left(1+\frac{b_{m_{0}, n}}{R(n)}\right) \cdot \prod_{i=0}^{d-1}\left(1-\frac{A_{m_{0}, i} \cdot b_{m_{0}, i}}{R(i)}\right) \\
& =\left(1+\frac{b_{m_{0}, n}}{R(n)}\right) \cdot \prod_{i=0}^{d-1}\left(1-R_{m_{0}, i}\right) .
\end{aligned}
$$

First, it is straightforward to show that

$$
\begin{aligned}
& 1+\frac{b_{m_{0}, n}}{R(n)} \\
\{b y(3)\} \geq & 1+\frac{B_{m_{0}, n}}{\frac{B(n)}{d(n)+1}-M B(n)} \\
\geq & 1+\frac{B_{m_{0}, n}}{\frac{B(n)}{d(n)+1}-B_{m_{0}, n}} \\
& =\frac{1}{1-R_{m_{0}, n}} .
\end{aligned}
$$

Next, using (10) and Lemma 1 (in Appendix A.), we can show

$$
\begin{aligned}
\prod_{i=0}^{d-1}\left(1-R_{m_{0}, i}\right) & \geq 1-\sum_{i=0}^{d-1} R_{m_{0}, i} \\
(b y(8)) & >1-R_{m_{0}, n}
\end{aligned}
$$

By combining (12) and (13) into (11), we get

$$
G_{F P}>\frac{1}{1-R_{m_{0}, n}} \cdot\left(1-R_{m_{0}, n}\right)=1
$$

The above results show that the system utility increases after the feeding, which contradicts with the assumption that assignment $A$ is FC-optimal.

Theorem 1 shows that the lower bound of each user's throughput depends on its interference condition. First, the bound of user $n$ scales inversely with the number of conflicting neighbors of $n$, that is, users get less spectrum in crowded areas than in sparse areas. This scaling provides an immediate intuition of fairness.

Second, the bound scales linearly with the node's total available throughput $B(n)$. If a user improves its channel bandwidth using sophisticated physical layer techniques, without changing its interference condition, the throughput bound increases linearly, but the number of assigned channels remains the same. Hence, the proposed strategy will not favor users in good channel conditions and starve users in bad channel conditions.

\section{B. Poverty Line on User Spectrum}

Under certain circumstances, channel quality fluctuates due to fading, shadowing and environmental factors, making it impractical to collect channel quality in real time. Hence, a reasonable metric to evaluate spectrum allocation is the number of spectrum channels assigned to a user, regardless of its bandwidth. Next we show that Theorem 1 can be reduced to the following:

Theorem 2: Under a FC-optimal assignment A, for each vertex $n$ in the conflict graph $G, 0 \leq n \leq N-1$ with degree $d(n)$ and channel availability list $L(n)$, its spectrum usage $R(n)$ has a lower bound, i.e.

$$
R(n) \geq\left\lfloor\frac{|L(n)|}{d(n)+1}\right\rfloor \triangleq P L(n) .
$$


Proof: By Theorem 1, when all channels have bandwidth 1 , we have

$$
R(n)>\frac{|L(n)|}{d(n)+1}-1
$$

Since $R(n)$ is an integer, this is equivalent to

$$
R(n) \geq\left\lfloor\frac{|L(n)|}{d(n)+1}\right\rfloor .
$$

Theorem 2 shows that the proposed Fairness Coordination with Feed Poverty guarantees a poverty line $P L(n)$ to each vertex $n$. The poverty line provides a guideline in coordination in real systems where a vertex is entitled to request coordination if its current throughput is below its poverty line. We refer to this as the poverty guided coordination.

Further, we can show that if channels are fully available at each vertex, i.e. $|L(n)|=M$, a FC-optimal assignment can eliminate user starvation if the number of channels $M \geq$ $\Delta+1$, where $\Delta$ is the maximum degree in the graph $\Delta=$ $\max _{0 \leq n<N} d(n)$. This matches to the well-known conclusion in graph coloring where the chromatic number of a graph is at most $\Delta+1$ [5].

Theorem 3: The poverty line bound in Theorem 2 is tight, for several types of network topology: clique, ring, star, and chain.

This can be shown by individual case studies, and hence we omit the proof due to space constraints. For randomly generated, clustered and real-network topologies, we obtain statistical results on the tightness of the bound using experiments in Section V-B.

\section{Upper Bound on Coordination Complexity}

Using Poverty guided coordination, only users with spectrum assignment less than their poverty line can initiate a coordination. The requestor $n$ can sequentially select the best channels that provide the highest improvement to system utility, and negotiate with the neighboring nodes to get these channels at one time. Following the detailed proof of Theorem $\mathbf{2}$, we can show that after each coordination iteration, $n$ 's spectrum assignment will reach its poverty line. This also allows us to derive an upper bound on the number of coordination iterations needed to reach a system equilibrium.

Theorem 4: In a system with $N$ nodes, with uniform channel bandwidth, the poverty guided coordination will reach an equilibrium after an expected number of at most $O\left(N^{2}\right)$ iterations. By optimizing the order of coordination, the system can reach an equilibrium in at most $N$ iterations.

Proof: We denote a user as "satisfied" if it is on or above its poverty line, otherwise "unsatisfied". First we present a key property of the poverty guided coordination (PGC):

Property 1: In a coordination iteration in which channels are feeded to $n$, if a neighbor $n_{1}$ is converted from "satisfied" to "unsatisfied", then $P L(n)<P L\left(n_{1}\right)$. (proof in Appendix B)

From property 1, if the PGC can be conducted such that the user with lower poverty line among its (one-hop) neighborhood wins the priority to be feeded first, then each "satisfied" user will never become an "unsatisfied" user after it is feeded by the PGC. Hence, the number of iterations is at most $N$. If the user to be feeded is selected randomly among requestors, then a user with lower poverty line among its neighborhood will wait for an expected number of $O(N)$ iterations to win the opportunity, because at each iteration the number of users who request a PGC is bounded by $N$. The total number of iterations until the system reaches an equilibrium is thus $O\left(N^{2}\right)$.

It should be noted that the $O\left(N^{2}\right)$ bound in Theorem 4 is a theoretical upper bound on the complexity of the coordination system, which does not necessarily mean that the complexity of the system will scale in a $O\left(N^{2}\right)$ trend in practice. Actually as we observed in our simulation (Section V-D), the complexity of the system scales linearly as the number of nodes increases.

\section{EXPERIMENTAL RESUlTS}

We conduct experimental simulations to quantify the performance of coordination-based spectrum allocation and validate the theoretical lower bounds. For default scenarios, we assume that channels are equally weighted and all the channels are available for each node, i.e. $l_{n, m}=1, b_{n, m}=1$. We assume that all active nodes have backlogged traffic.

We assume a network of many WiFi and WiMAX access points, referred to as users. We assume each AP serves a large number of subscribers and have backlogged traffic. We use a simple binary interference model to construct the conflict graph - two users conflict if they are within distance of $D$. By default, we set $D$ to $100 \mathrm{~m}$. We use this assumption to simplify the conflict graph construction. However, it will not limit the scope of the proposed coordination framework. To examine the impact of network topology, we use both randomly generated topologies and measured AP deployment traces.

- Random network. We place users randomly in an area.

- Clustered network. We simulate a hotspot scenario by deploying a set of users densely in a small area of the random network. We use this topology to examine the impact of conflict degree on system performance.

- Real network trace. We extract a set of actual AP deployments using data traces collected by Placelab (http://www.placelab.org/).

In selected scenarios, we randomly deploy primary users and introduce dynamics to spectrum availability at each node. We also introduce heterogeneous channel bandwidth and examine the impact on the tightness of poverty line. We use these simple scenarios to demonstrate the impact of network dynamics and examine the system convergence time and coordination overhead.

We use two metrics to evaluate the performance.

System utility - We consider fairness defined in (1). Note that if there exists a user with no channel assigned, the utility becomes $-\infty$. For better representation, we modify the utility 
to $U(A)=\sqrt[N]{\prod_{n=0}^{N-1} R_{A}(n)}$ and $U(A)=0$ if there is any $R_{A}(n)=0$.

Communication overhead - We quantify algorithm complexity as the communication overhead, i.e. total number of messages exchanged among nodes, since transmission and handling of messages will likely dominate computations for channel assignment. In both distributed coordination and graph coloring approaches, each iteration of spectrum assignment or coordination involves a 4-way handshake between neighbors, i.e. (request, acknowledgement, action, acknowledgement).

\section{A. Comparison with Centralized Graph Coloring Approach}

We compare the proposed distributed coordination to the centralized graph-coloring approach of [18]. We randomly deploy 40 links with 30 channels in a given area and produce the corresponding conflict graph. In this case, the proposed distributed coordination scheme starts from a random allocation and gradually improves system utility until the system converges. Figure 4 compares the system utility and algorithm complexity using centralized graph coloring, distributed coordination and random assignment. Random assignments results in frequent user starvation and hence zero system utility. Distributed coordination can effectively eliminate user starvation and performs only slightly worse compared to graph coloring approach. However, Figure 4(b) shows that distributed coordination can significantly reduce communication overhead, by 8 times for this configuration.

\section{B. Tightness of the Poverty Line Bound}

We now examine the appropriateness of the user poverty bound derived in Theorem 2 .

(1) Static Networks - Figure 5 illustrates the histogram of the ratio of the actual user throughput and the poverty bound assuming 40 vertices and 100 time slots. Results show that the theoretical bound is valid and fairly tight. As we described, nodes can use the poverty line to decide whether further distributed coordination is necessary. A vertex with an assignment below the poverty line should bargain with additional neighbors to acquire additional channels.

(2) Dynamic Spectrum Availability - Next, we examine the impact of primary user deployment on the bound. We randomly deploy 10 and 30 primary users to our previous experiments. Primary users have the same interference range as secondary users, and occupy one randomly chosen channel. Figure 6 shows the CDF of the ratio between actual node throughput and lower bound. We observe that the bound becomes slightly looser as the number of primary users increases. This is mainly due to the mismatch between $L(n)$ and $d(n)$. We calculate $d(n)$ to include all the interfering neighbors who has at least one channel in common with $n$. Therefore, $d(n)$ is the upper bound of the conflicting neighbors on each channel.

(3) Heterogenous Spectrum Bandwidth - When channel bandwidth is non-uniform, we compute the bound following Theorem 1. Figure 7 compares the tightness of the bound when channel bandwidth varies between 1 to 3 and 1 to 5 . The result

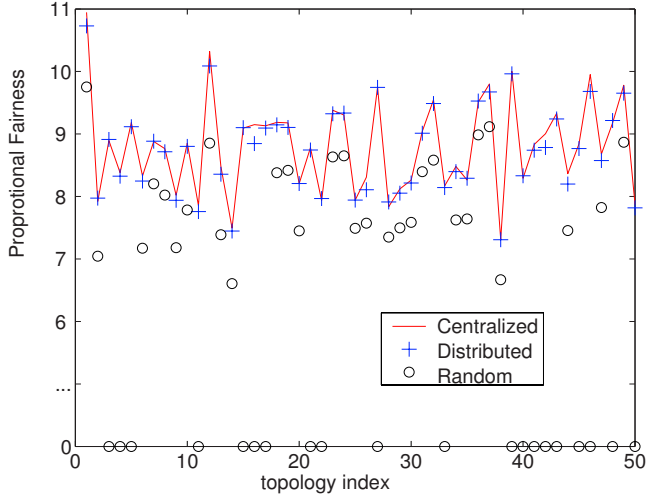

(a) System Utility

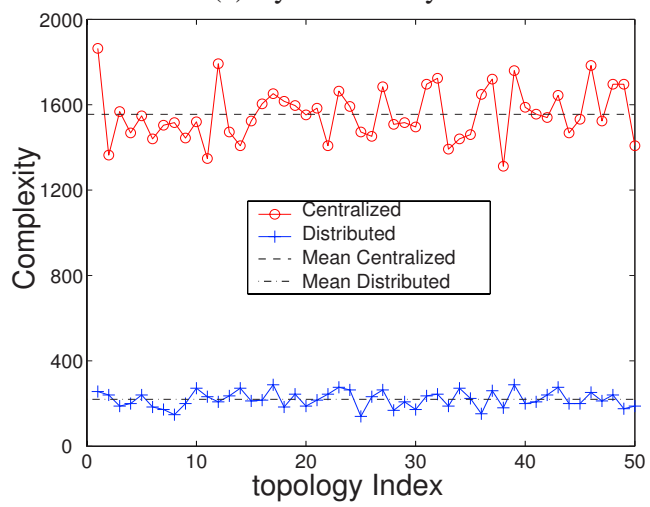

(b) Algorithm Complexity

Fig. 4. Performance comparison of centralized graph coloring, distributed coordination and random spectrum assignment for various topologies [4].

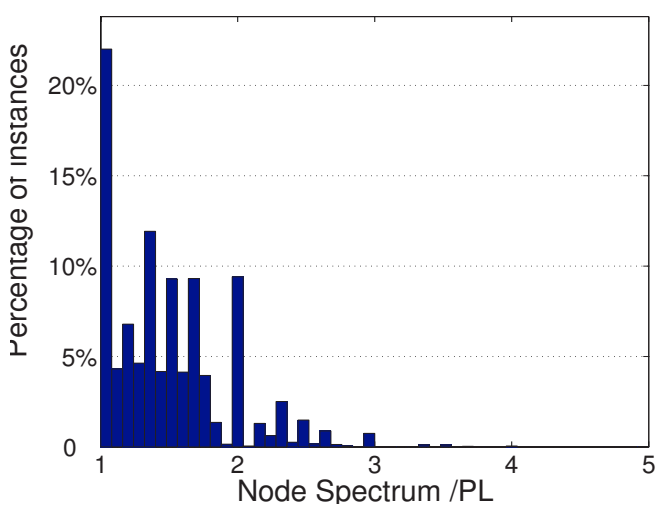

Fig. 5. Histogram of the ratio between the actual user throughput and the poverty bound.

is relatively smoother by eliminating the truncation effect in Theorem 2. We see that the bound is looser compared to that of the uniformed bandwidth. This is mainly due to the fact that a node's throughput is always strictly larger than the bound. The figure also shows that the bound is sensitive to the variance in bandwidth across channels.

\section{Impact of Network Topology}

Figure 8(a) illustrates three sample topologies corresponding to random, clustered and measured networks, respectively. 


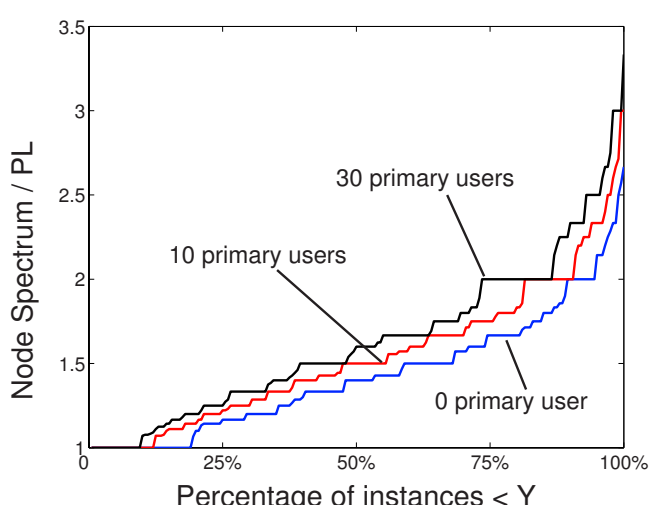

Fig. 6. Tightness of the Poverty Line bound when there are primary users.

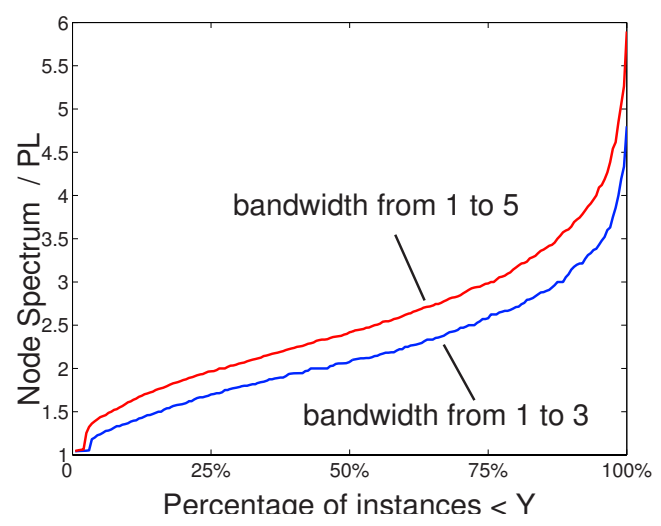

Fig. 7. Tightness of the Poverty Line Bound under heterogeneous channel bandwidth.

Every topology contains 200 nodes, and the number of channels is 100 . We use 100 channels since most nodes in clustered and measured networks have degree larger than 30 . Note that for this experiment the initial allocation is the empty allocation. Figure 8(b) plots the amount of spectrum assigned to each user using the explicit coordination. We observe that any user $n$ obtains spectrum inversely proportional to its conflict degree $d(n)$, as characterized by the poverty line definition.

To further investigate this dependency, we plot in Figure 8(c) the amount of spectrum assigned at each user divided by its poverty line, as a function of the conflict degree $d(n)$. It can be observed that in the clustered network and the measured network, there are three kinds of users: 1) the users in the non-clustered area, which is consistent with the nodes in the random network; 2) the users at the clustered area, whose degrees are high; 3 ) the users near the edge of the clustered area, whose conflict degrees are between that of the clustered area and the non-clustered area (around 10-20). The results show that the Poverty Line bound is relatively tighter for the first two types of users, and becomes looser for the third type. In other words, the Poverty Line bound is more accurate for homogenous conflict conditions, which coincides with the theoretical results where the Poverty Line is tight for the line, clique, and ring topologies.

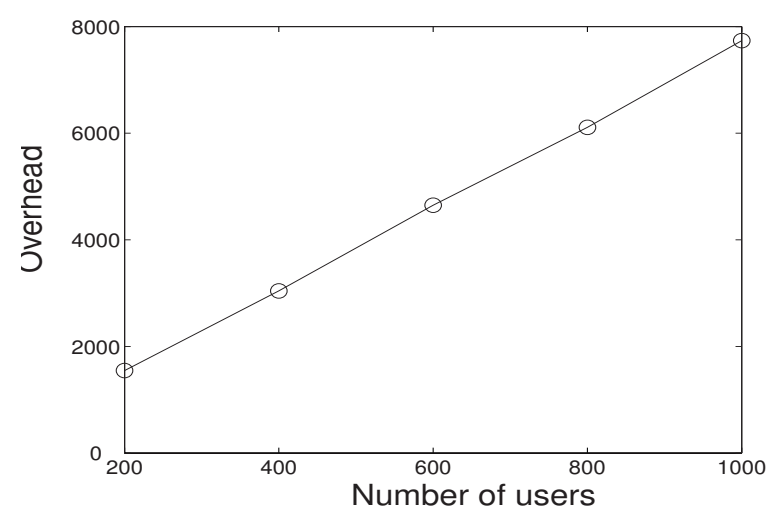

Fig. 9. System overhead in large scale networks

Figure 8(d) shows the communication overhead at each user as a function of the conflict degree $d(n)$. In the clustered network, the results show that the users at the edge of the cluster have more communication overhead. Further, we also observe that although the total complexity scales linearly with the network size, it also scales with the number of channels. However, we could not obtain the relationship of the complexity and the number of channels. We plan to study the individual complexity in a future work.

\section{Scalability of Distributed Coordination}

We evaluate the complexity of coordination in large scale networks. We measure the total system overhead, i.e. the total number of message exchanges for a system to reach an equilibrium. We keep the user density constant and vary the system scale from 200 to 1000 , and assume $20 \%$ of node dynamics, and 20 channels. Results in Figure 9 indicates that the system overhead (in a random network) scales linearly with the number of users. Hence, the average overhead per user is roughly constant, 8 messages or 2 coordination iterations under the above system configurations. This result demonstrates the efficiency of the proposed approach. We note that the per-user overhead is much smaller in this scenario than that in Figure 8. This is because we use less number of channels.

\section{CONClusion And Future Work}

In this paper, we study the performance of an adaptive and distributed approach for spectrum sharing in dynamic spectrum networks. In this approach, users self-organize into coordination groups and adapt their spectrum assignment to approximate the global optimal assignment. We perform analytical study on each user's spectrum assignment and coordination overhead. We derive a lower bound on each user's spectrum usage guaranteed by the coordination, referred to as the poverty line. It represents the level of fairness enforced by the coordination, and serves as a guideline to organize coordination. Experimental results show that the proposed approach performs similarly as the centralized topologyoptimized approach but with much less complexity. We also verify the correctness of the poverty line under various network 
settings. While we only proposed a specific coordination strategy to maximize fairness based system utility, the proposed coordination framework can be extended towards other utility functions or optimization goal. We intend to examine this in a future study.

\section{REFERENCES}

[1] Berger, R. J. Open spectrum: a path to ubiquitous connectivity. ACM Quеue 1, 3 (May 2003).

[2] Buddhikot, M. M., Kolody, P., Miller, S., Ryan, K., ANd EVANS, J. DIMSUMNet: New directions inwireless networking using coordinated dynamic spectrum access. In Proc. of IEEE WoWMoM (June 2005).

[3] Buddhikot, M. M., And Ryan, K. Spectrum management in coordinated dynamic spectrum access based cellular networks. In Proc. of IEEE DYSPAN (November 2005).

[4] CAO, L., AND Zheng, H. Spectrum allocation in ad hoc networks via local bargaining. In Proc. of SECON (September 2005).

[5] Chartrand, G. A Scheduling Problem: An Introduction to Chromatic Numbers. New York: Dover, pp.202-209, 1985.

[6] Clemens, N., And Rose, C. Intelligent power allocation strategies in an unlicensed spectrum. In Proc. of IEEE DySPAN (November 2005).

[7] Garey, M. R., And Johnson, D. S. Computers and Intractability: A Guide to the Theory of NP-Completeness. W. H. Freeman, 1990.

[8] HAYKIN, S. Cognitive radio: Brain-empowered wireless communications. IEEE Journal on Selected Areas in Communications 23, 2 (Feburary 2005), 201-220.

[9] HuAng, J., BERry, R., AND Honig, M. Auction mechanisms for distributed spectrum sharing. In Proc. of 42nd Allerton Conference (September 2004)

[10] Huang, J., Berry, R. A., And Honig, M. L. Spectrum sharing with distributed interference compensation. In Proc. of IEEE DySPAN (November 2005).

[11] Ileri, O., Samardzija, D., And Mandayam, N. B. Demand responsive pricing and competitive spectrum allocation via spectrum server. In Proc. of IEEE DySPAN (November 2005).

[12] Jain, K., Padhye, J., V.N.Padmanabha, And QiU, L. Impact of interference on multi-hop wireless network performance. In Proc. of MobiCom (September 2003).

[13] Jones, S. D., Merheb, N., And Wang, I.-J. An experiment for sensing-based opportunistic spectrum access in CSMA/CA networks. In Proc. of IEEE DySPAN (Baltimore, MD, Nov 2005)

[14] Katzela, I., AND Naghshineh, M. Channel assignment schems for celluar mobile telecommunication systems. IEEE Personal Communications 3, 3 (June 1996), 10-31.

[15] Mitola III, J. Wireless architectures for the 21st century. http: //ourworld.compuserve.com/homepages/jmitola.

[16] NeEl, J., Reed, J., AND Gilles, R. The role of game theory in the analysis of software radio networks. In Proc. Software Defined Radio Forum Technical Conference and Product Exhibition (SDR 02) (San Diego, CA, USA, November 2002), vol. 2, pp. pp. NP-3-02.

[17] NiE, N., AND ComaniciU, C. Adaptive channel allocation spectrum etiquette for cognitive radio networks. In Proc. of IEEE DySPAN (November 2005).

[18] Peng, C., Zheng, H., And Zhao, B. Y. Utilization and fairness in spectrum assignemnt for opportunistic spectrum access. Mobile Networks and Applications (MONET) 11 (May 2006), 555-576.

[19] Raman, C., Yates, R., And Mandayam, N. Scheduling variable rate links via a spectrum server. In Proc. of IEEE DySPAN (November 2005).

[20] Ramanathan, S., AND Lloyd, E. Scheduling algorithms for multihop radio networks. IEEE/ACM Transactions on Networking 1, 2 (April 1993), 166-177.

[21] Ryan, K., Aravantinos, E., And Buddhikot, M. M. A new pricing model for next generation spectrum access. In Proc. of TAPAS (August 2006).

[22] Sankaranarayanan, S., Papadimitratos, P., Mishra, A., And HERSHEY, S. A bandwidth sharing approach to improve licensed spectrum utilization. In Proc. of IEEE DySPAN (November 2005).

[23] Zheng, H., AND PENG, C. Collaboration and fairness in opportunistic spectrum access. In Proc. of ICC (June 2005).

\section{APPENDIX}

\section{A. Lemma 1 and Its Proof}

Lemma 1: Suppose $a_{1}, a_{2}, \cdots, a_{n} \geq 0$, and $a_{1}+a_{2}+\cdots+$ $a_{n}=p<1$. Let $f\left(a_{1}, a_{2}, \cdots, a_{n}\right)=\left(1-a_{1}\right)\left(1-a_{2}\right) \cdots(1-$ $\left.a_{n}\right)$. Then $f\left(a_{1}, a_{2}, \cdots, a_{n}\right) \geq 1-p$.

Proof: $f$ is minimized when there is only one non-zero number, i.e. $a_{i}=p$ and $a_{j}=0$ for $j \neq i$. To prove this, let's assume that there are two non-zero numbers, i.e. $a_{i} \neq 0$ and $a_{j} \neq 0$. We modify $a_{i}^{\prime}=a_{i}+a_{j}$ and $a_{j}^{\prime}=0$, so $a_{i}+a_{j}=$ $a_{i}^{\prime}+a_{j}^{\prime}$. However,

$\left(1-a_{i}^{\prime}\right)\left(1-a_{j}^{\prime}\right)=1-a_{i}-a_{j}<1-a_{i}-a_{j}+a_{i} a_{j}=\left(1-a_{i}\right)\left(1-a_{j}\right)$.

Thus, when there are more than one non-zero numbers in $a_{i}, 1 \leq i \leq n$, we can modify the $a_{i}$ s to reduce $f$. This shows that $f$ is minimized by having only one positive $a_{i}$, and $f=1-p$.

\section{B. Proof of Property 1}

Proof: First we assume node $n$ is below its poverty line $P L(n)$. From the proof of Theorem 1, by combining (11), (12) and (14), we show that for channel $m_{0}$,

$$
\frac{1}{1-R_{m_{0}, n}} \cdot \prod_{i=0}^{d-1}\left(1-R_{m_{0}, i}\right)>1 .
$$

Under uniform channel bandwidth, this shows that before the coordination,

$$
\left(\frac{1}{1-\frac{d+1}{|L(n)|}}\right) \cdot \prod_{i=0}^{d-1}\left(1-\frac{1}{R(i)}\right)>1 .
$$

Since $\left(1-\frac{1}{R(i)}\right)<1$ and $\frac{d+1}{|L(n)|} \leq \frac{1}{P L(n)}$, this implies that for any neighbor $n_{1}, 0 \leq n_{1} \leq d-1$ involved in the coordination,

$$
\frac{P L(n)}{P L(n)-1} \cdot \frac{R\left(n_{1}\right)-1}{R\left(n_{1}\right)}>1
$$

and therefore, $R\left(n_{1}\right)>P L(n)$. Hence, after feeding channel $m_{0}$ to $n$, no neighbor's throughput is reduced to below $P L(n)$. Hence, if the throughput of $n_{1}$ is reduced to below $P L\left(n_{1}\right)$, then it must be the case where $P L(n)<P L\left(n_{1}\right)$. 

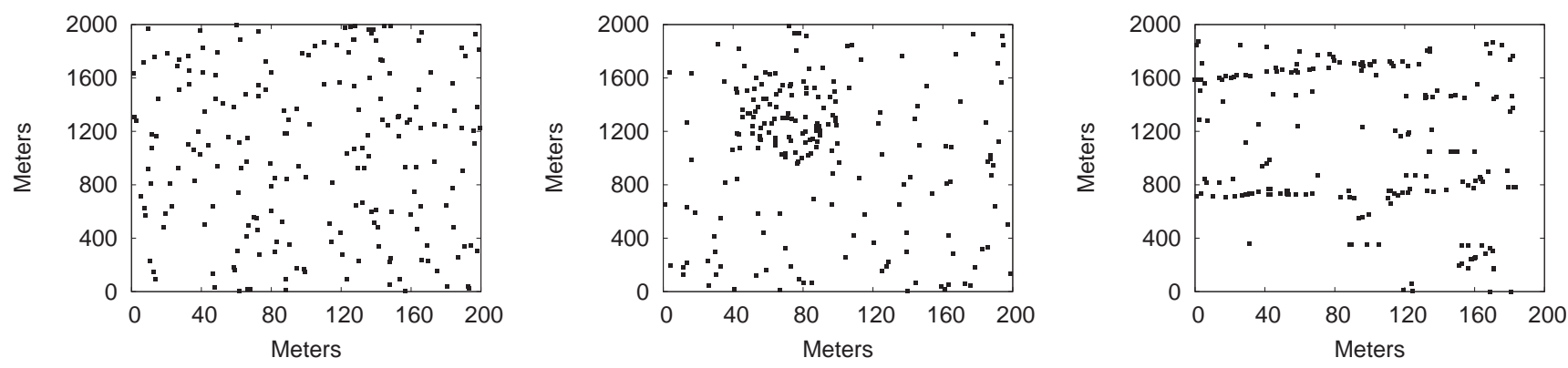

(a) Network topology

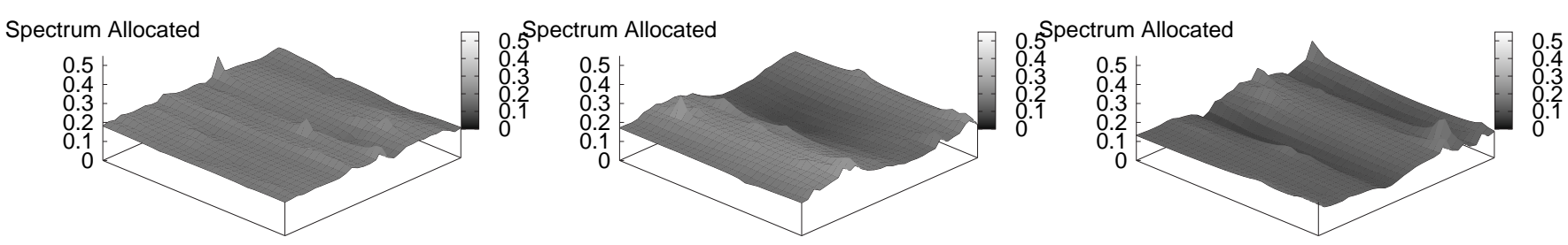

(b) The amount of spectrum assigned to each node.
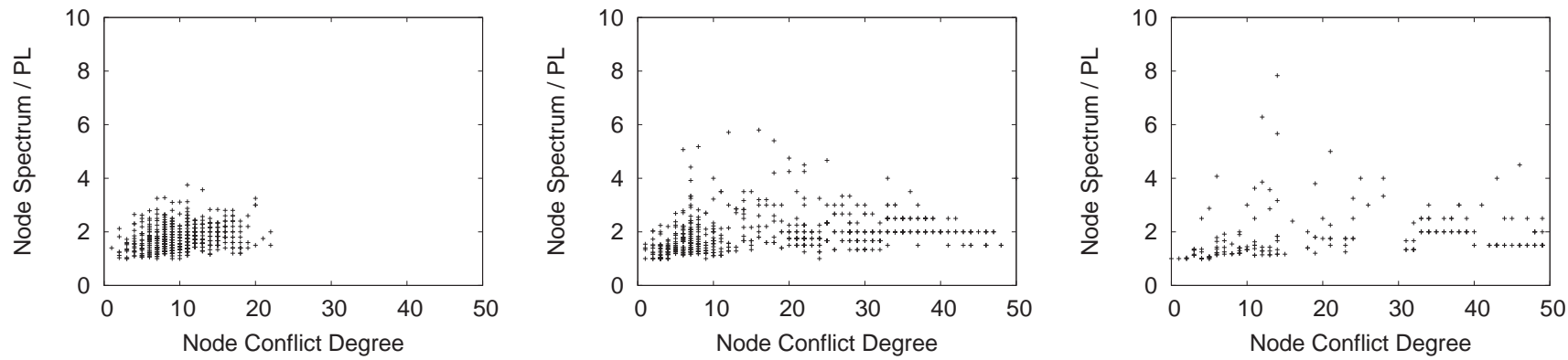

(c) The tightness of Poverty Line bound.
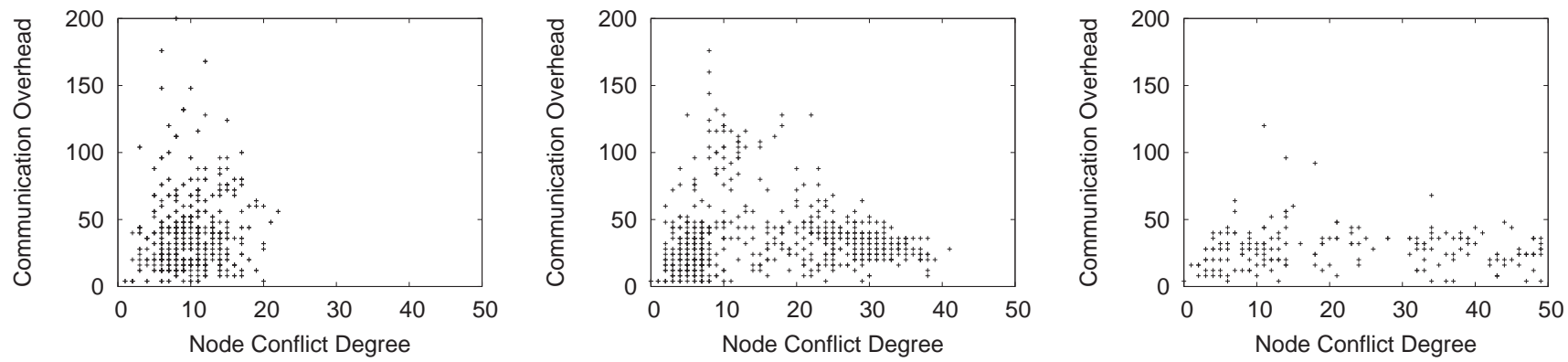

(d) The communication overhead.

Fig. 8. The amount of spectrum assigned to each node for (left) random network, (center) clustered network and (right) placelab network. 